\title{
Small calcified lesions suggestive of neurocysticercosis are associated with mesial temporal sclerosis
}

\section{Pequenas lesões calcificadas sugestivas de neurocisticercose e estão associadas com esclerose mesial temporal}

Marcos C. B. Oliveira ${ }^{1}$, Maria G. M. Martin ${ }^{2}$, Miriam H. Tsunemi ${ }^{3}$, Gilson Vieira ${ }^{4}$, Luiz H. M. Castro ${ }^{1}$

\begin{abstract}
Recent studies have suggested a possible relationship between temporal lobe epilepsy with mesial temporal sclerosis (MTS) and neurocysticercosis (NC). We performed a case-control study to evaluate the association of NC and MTS. Method: : We randomly selected patients with different epilepsy types, including: MTS, primary generalized epilepsy (PGE) and focal symptomatic epilepsy (FSE). Patients underwent a structured interview, followed by head computed tomography (CT). A neuroradiologist evaluated the scan for presence of calcified lesions suggestive of NC. CT results were matched with patients' data. Results: : More patients in the MTS group displayed calcified lesions suggestive of NC than patients in the other groups $(p=0.002)$. On multivariate analysis, MTS was found to be an independent predictor of one or more calcified NC lesions ( $p=0.033$ ). Conclusion: : After controlling for confounding factors, we found an independent association between NC calcified lesions and MTS.
\end{abstract}

Keywords: hippocampal sclerosis, cysticercosis, temporal lobe epilepsy, mesial temporal sclerosis, neurocysticercosis, calcifications.

\section{RESUMO}

Estudos recentes têm sugerido possível relação entre epilepsia do lobo temporal com esclerose mesial temporal (MTS) e neurocisticercose (NC). Conduzimos um estudo caso-controle para avaliar a associação de NC e MTS. Método: Selecionamos randomicamente pacientes com diferentes tipos de epilepsia, incluindo: MTS, epilepsia primariamente generalizada (PGE) e epilepsia focal sintomática (FSE). Pacientes foram submetidos a uma entrevista estruturada, seguida por uma tomografia computadorizada de crânio (CT). Um neuroradiologista avaliou as imagens quanto à presença de lesões calcificadas sugestivas de NC. Resultados das CT foram comparados com os dados dos pacientes. Resultados: Mais pacientes do grupo MTS apresentaram lesões calcificadas sugestivas de NC que de outros grupos ( $p=0,002)$. Na análise multivariável, MTS foi um preditor independente de uma ou mais calcificações sugestivas de NC ( $p=0,033)$. Conclusão: Após controlar para fatores confusionais, encontramos uma associação independente entre lesões calcificadas de NC e MTS.

Palavras-chave: esclerose de hipocampo, cisticercose, epilepsia do lobo temporal, esclerose mesial temporal, neurocisticercose, calcificações.

Temporal lobe epilepsy associated with mesial temporal sclerosis (MTS) is the most common cause of medically refractory epilepsy in adults ${ }^{1}$. The cause of MTS remains incompletely understood. Genetic factors play a role in familial cases ${ }^{2}$. In other cases, there is an association with an initial precipitating insult (IPI), more commonly a complicated febrile seizure occurring before three years of age ${ }^{1}$.
Neurocysticercosis (NC) results from brain invasion with Taenia solium. Radiologic features of the chronic form consist of small punctate calcifications easily seen on brain imaging. Seizures in NC occur mainly in association with degenerating cysts (acute symptomatic seizures) ${ }^{3,4}$. Active epilepsy, especially refractory epilepsy, appears to be less common in $\mathrm{NC}^{3,4}$.

\footnotetext{
'Departamento de Neurologia, Faculdade de Medicina, Universidade de São Paulo, Sao Paulo SP, Brazil;

${ }^{2}$ Laboratório de Investigações Médicas 44, Instituto de Radiologia, Hospital das Clínicas, Faculdade de Medicina de São Paulo, Sao Paulo SP Brazil;

${ }^{3}$ Departamento de Bioestatística, Instituto de Biociências, Campus de Botucatu Distrito de Rubião Júnior, Universidade Estadual Paulista “Júlio de Mesquita Filho", Botucatu SP, Brazil;

${ }^{4}$ Instituto de Radiologia, Hospital das Clínicas, Faculdade de Medicina de São Paulo, Sao Paulo SP, Brazil.

Correspondence: Marcos C. B. Oliveira; Av. Dr. Enéas de Carvalho Aguiar, 255 / $5^{\circ}$ andar / sala 501; 05403-900 São Paulo SP, Brasil;

E-mail: marcoscbo@hotmail.com

Conflict of interest: There is no conflict of interest to declare.

Support: The State of Sao Paulo, Brazil, Research Foundation (FAPESP), a state government agency sponsored this study through grants to M.C.B.O (Grant 2008/04119-4) and CInAPCe Program (Grant 2005/56464-9). The sponsor had no involvement in study design, collection, analysis and interpretation of data; in the writing of the report; and in the decision to submit the paper for publication.

Received 16 April 2014; Accepted 13 May 2014.
} 
Small calcified lesions suggestive of inactive NC are commonly seen in asymptomatic individuals living in endemic regions. The presence of inactive NC lesions in patients with epilepsy does not appear to influence clinical features, surgical outcome or hippocampal histological findings, suggesting that NC may represent an incidental finding in this setting ${ }^{5}$. Inactive NC is seen in up to $38 \%$ of epilepsy patients with MTS undergoing presurgical evaluation ${ }^{6}$. Based on increased prevalence of inactive NC in MTS patients, a possible causal relationship between $\mathrm{NC}$ and MTS has been proposed ${ }^{6}$. The authors speculated that inflammatory lesions or repetitive seizures might play a role in disease pathogenesis ${ }^{6,7}$.

Isolated case reports and small case series have suggested a possible association between $\mathrm{NC}$ and mesial temporal sclerosis, in which typical mesial temporal sclerosis developed after cysticercosis-related acute symptomatic seizures ${ }^{8,9,10}$.

Two case-control studies evaluated the prevalence of NC in refractory epilepsy patients ${ }^{11,12}$. One study that evaluated 512 patients with medically refractory epilepsy found a higher prevalence of $\mathrm{NC}$ in patients with epilepsy associated with MTS than in patients with epilepsy of other etiologies ${ }^{11}$. This study is limited by the lack of control for possible confounding factors, such as socioeconomic status or exposure to pigs. Another study did not find differences in frequency of hippocampal atrophy, studying 89 patients with and without $\mathrm{NC}^{12}$.

We performed a case control study to evaluate the association of NC and MTS, controlling for possible confounding factors.

\section{METHOD}

\section{Patient selection and epilepsy classification}

Study participants were identified in the adult epilepsy outpatient clinic in our institution, a major tertiary referral center and academic medical center in a large metropolitan region in Southeastern Brazil.

We identified, through chart review, according to the clinic schedule for follow-up appointments, consecutive cases of the following epilepsy groups:

(i) Focal symptomatic temporal lobe epilepsy associated with mesial temporal sclerosis;

(ii) Primary generalized epilepsy (PGE);

(iii) Focal symptomatic epilepsy, excluding MTS (FSE).

Epilepsy diagnosis was based on clinical, EEG and brain imaging findings. All patients in group one had previously undergone a brain MRI; all patients in group two and three had undergone a brain imaging study (CT or MRI). In group two diagnosis was established by typical clinical and EEG findings, consistent with the diagnosis of primary generalized epilepsy. We did not include individuals with dual pathology and patients who had undergone epilepsy surgery.

Cases were not excluded from the study if there was a previous diagnosis of neurocysticercosis or brain calcified lesions. In this study, diagnosis and epilepsy classification in cases with calcified lesions did not take into account the presence of calcified lesions; i.e. a case with one or more calcified lesions and clinical and EEG findings of myoclonic juvenile epilepsy would be classified as PGE. Cases with active cysticercosis (intact or degenerating cysts) were not included in the study.

If willing to participate, the patient signed the Institutional Review Board (IRB) - approved informed consent form (Cappesq - Process 0141/08). The patient then underwent a structured interview, to collect demographic data, epilepsy features, treatment and $\mathrm{NC}$ epidemiological data. After this interview, medical records data were also analyzed to complement patient information.

\section{Demographics}

Demographic data included age, gender, education years, and socioeconomic (SE) status. Patients were classified in one of five SE classes, based on a socioeconomic questionnaire ${ }^{13}$. Epilepsy features consisted of: epilepsy group, seizure features, age at onset, epilepsy duration, occurrence of an initial precipitating insult, family history of epilepsy, EEG, and brain imaging results. Treatment data included antiepileptic drug type, number and doses, and number of seizures in the preceding three months. Epidemiologic data consisted of a history of having lived in rural areas and of previous contact with pigs.

\section{Head CT}

After undergoing the structured interview, all patients were given a brain CT appointment within one week. All patients underwent the same CT protocol. Head CTs were systematically analyzed by the same neuroradiologist (MGMM), blinded to clinical, EEG, MRI and epilepsy syndrome data. Presence of small punctate calcified lesions suggestive of inactive NC was noted. We considered all intraparenchymal round calcified lesions with less than 10 $\mathrm{mm}$ in diameter, without changes in the adjacent brain parenchyma, as suggestive of neurocysticercosis. Meningeal and pallidal lesions, as well as tumoral calcifications, were not considered. Number of lesions and location were also evaluated. Cases were classified as containing no, one or two or more calcified lesions.

After a CT report had been completed for all cases, a different author (MCBO) matched results to epilepsy group (PGE, MTS and FSE), and compared the frequency of calcified lesions in the different epilepsy groups. We then 
compared demographic data, epilepsy features, treatment, and NC epidemiologic data between NC-positive and NCnegative MTS cases.

In order to test for a possible selection bias, we also compared demographic data, epilepsy features, treatment and NC epidemiologic data between patients who underwent a head CT, and those who did not show up for the CT appointment.

\section{Statistical analysis}

We used univariate analysis to compare the three groups for prevalence of small-calcified lesions (one or more, and two or more lesions), demographic data, epilepsy features, treatment and NC epidemiologic data. We also compared data between calcification-positive (one or more) and calcification-negative MTS cases, as well as for patients who underwent a head CT and those who did not show up for the CT appointment.

Logistic regression (multivariate analysis) was performed, considering as the independent variable the presence of two or more calcifications, and repeated with one or more calcifications. Significance level was defined as $p<0.05$.

\section{RESULTS}

\section{Patient selection}

One hundred sixty one patients signed the informed consent form, completed the structured interview and received a head CT appointment. Of these, 119 patients underwent a head CT (Figure).

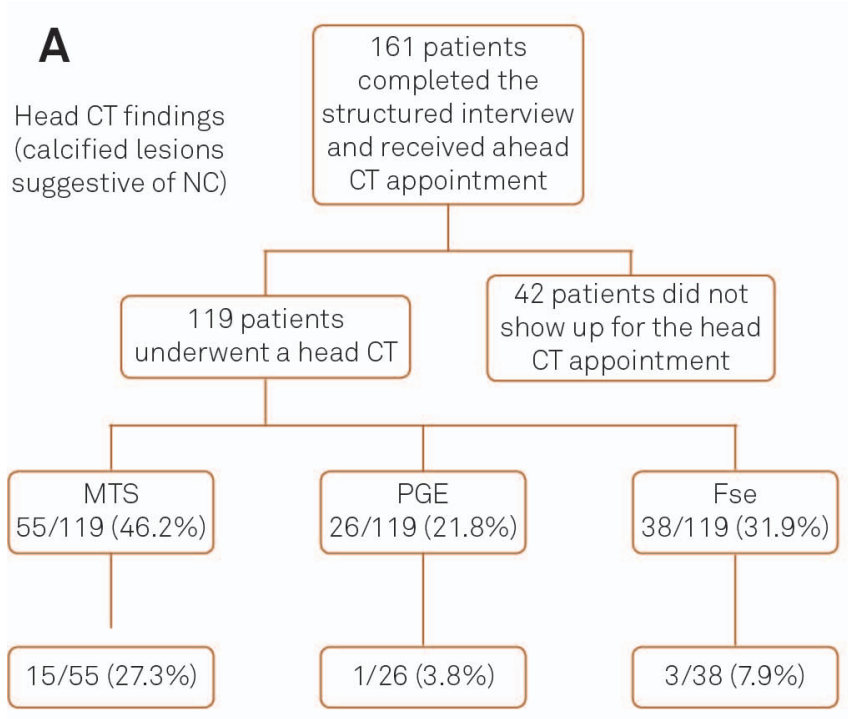

All patients had undergone previous brain imaging studies; 111/119 (93.3\%) patients had a previous brain MRI, the remaining had a previous head CT. All patients in the MTS group had previously undergone a brain MRI. In eight patients brain calcified lesions had been previously seen on head MRI or CT. Of these, three had the diagnosis of neurocysticercosis documented on their charts. In eleven patients one or more calcified lesions were only identified in the head CT performed for this study.

Patients who underwent a head CT and patients who did not show up for the CT appointment did not differ in terms of epilepsy group, gender, education, socioeconomic status, onset and duration of epilepsy, number of medications, and NC epidemiology. Patients who underwent a head CT were older ( $40.6 \pm 11.9$ vs. $36.4 \pm 12.9$ years; $p=0.049$ ), and had more seizures in the preceding three months $(6.7 \pm 17.0$ vs. $1.6 \pm 2.9$ seizures; $\mathrm{p}=0.025)$, compared to patients that did not show up for the CT appointment.

\section{Head CT findings}

A greater proportion of patients in MTS group had head CT findings of calcified lesions suggestive of inactive neurocysticercosis than the other groups, considering presence of one or more lesions ( $15 / 55$ or $27.3 \%$ vs. $4 / 64$ or $6.2 \%$; $\mathrm{p}=0.002)$, and of two or more lesions $(11 / 55$ or $20.0 \%$ vs. $2 / 64$ or $3.1 \%$; $=0.003$ ). (Table 1 )

Calcified lesions suggestive of $\mathrm{NC}$ were found in 15 patients in the MTS group (four patients had one lesion, seven patients had three or more lesions), and in four patients of the other groups (two patients with one lesion, one patient with two lesions and one patient with four

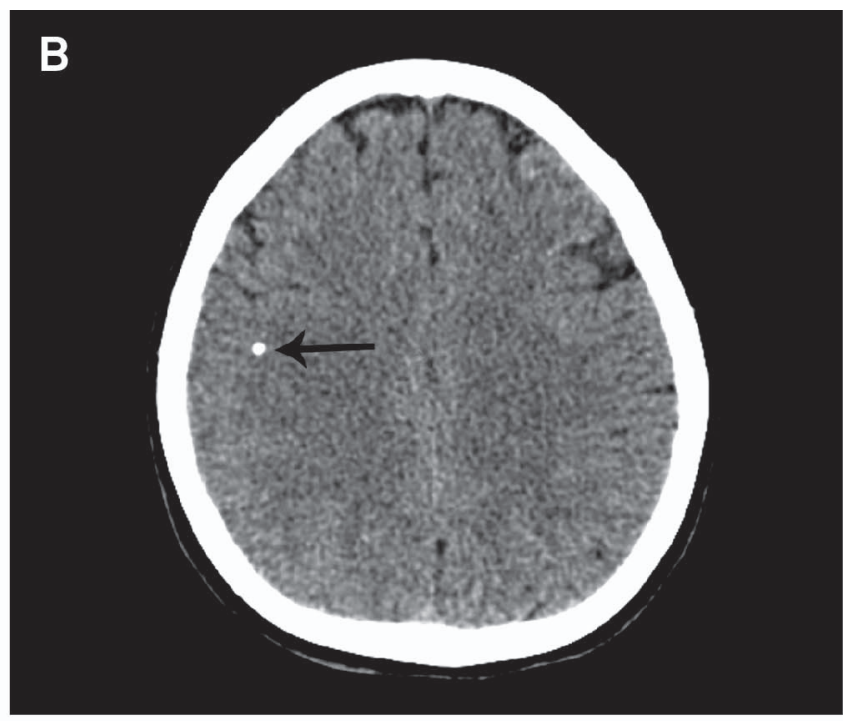

MTS: Mesial temporal sclerosis; PGE: Primary generalized epilepsy; FSE: Focal symptomatic epilepsy; FCE: Nontemporal focal cryptogenic epilepsy; NC: Neurocysticercosis.

Figure. Patient selection and head CT findings. 
Table 1. Head CT findings and NC epidemiology for different epilepsy types (MTS compared to PGE, FSE and FCE).

\begin{tabular}{|c|c|c|c|c|}
\hline & $\begin{array}{c}\text { MTS } \\
(n=55)\end{array}$ & $\begin{array}{c}\text { PGE } \\
(n=26)\end{array}$ & $\begin{array}{c}\text { FSE } \\
(n=38)\end{array}$ & $p$-value* \\
\hline Presence of calcified lesions suggestive of NC (one or more) & $15(27.3 \%)$ & $1(3.8 \%)$ & $3(7.9 \%)$ & 0.002 \\
\hline Presence of calcified lesions suggestive of NC (two or more) & $11(20.0 \%)$ & $0(0.0 \%)$ & $2(5.3 \%)$ & 0.003 \\
\hline History of having lived in rural areas & $34(61.8 \%)$ & $6(23.1 \%)$ & $11(28.9 \%)$ & $<0.001$ \\
\hline Previous contact with pigs & $33(60.0 \%)$ & $7(26.9 \%)$ & $15(39.5 \%)$ & 0.005 \\
\hline
\end{tabular}

MTS: Mesial temporal sclerosis group; PGE: Primary generalized epilepsy group; FSE: Focal symptomatic epilepsy group; NC: Neurocysticercosis; N: number of cases. *Between group statistical comparison: MTS vs. FSE and PGE.

lesions). The average number of calcified lesions was 5.4 in the MTS group and 2.0 in the other groups. Nine patients had calcifications in the temporal lobe (seven in the MTS group, six of which on the same side as the hippocampal sclerosis).

No active NC lesions or racemose cysts were seen on patients' CT. PGE and FSE groups did not differ in terms of presence of calcified lesions on head CT.

\section{Multivariate analysis}

Controlling for possible confounding factors with multivariate analysis, the presence of one or more calcified lesions was independently associated with the finding of mesial temporal sclerosis $(p=0.033)$. The association between presence of two or more calcified lesions and the finding of mesial temporal sclerosis did not reach statistical significance $(p=0.08)$. Presence of one or more calcified lesions was independently associated with a history of previous contact with pigs $(\mathrm{p}=0.011)$, as was presence of two or more calcifications $(p=0.018)$.

\section{Demographics and epilepsy features}

On univariate analysis, MTS patients more frequently referred a history of having lived in rural areas and of having had contact with pigs (Table 1). Data comparing demographics and epilepsy features of the three groups is detailed on Table 2.

\section{Comparison between MTS cases with and without calcified lesions}

MTS patients who displayed one or more calcified lesions on head CT more frequently referred a previous contact with pigs, a history of having lived in rural areas, and longer epilepsy duration than MTS patients without NC calcifications on CT (Table 3). On multivariate analysis, previous contact with pigs was the only independent predictor of $\mathrm{NC}$ in MTS patients $(p=0.013$; OR=15.3). The groups did not differ in terms of occurrence of an initial precipitating insult.

\section{DISCUSSION}

In order to assess a possible association between NC and MTS the ideal study format would be a population-based study of the frequency of calcified lesions in the general population, and in different epilepsy syndromes, including focal symptomatic epilepsy secondary to MTS. These studies would require MRI studies, which may not be readily available in endemic regions. Preliminary studies, investigating this possible association, in a case-control format, controlling for possible confounding factors, are therefore highly desirable to evaluate a possible association between small calcified lesions and MTS.

A few observational studies, including this one, have encountered a higher prevalence of calcified lesions

Table 2. Between group comparisons of epilepsy features for the different epilepsy groups.

\begin{tabular}{|c|c|c|c|c|}
\hline & $\begin{array}{c}\text { MTS } \\
(n=55)\end{array}$ & $\begin{array}{c}\text { PGE } \\
(n=26)\end{array}$ & $\begin{array}{c}\text { FSE } \\
(n=38)\end{array}$ & p-value \\
\hline Age & $43.4 \pm 12.1$ & $34.9 \pm 9.8$ & $40.4 \pm 11.9$ & $0.002^{b}$ \\
\hline Education (years) & $7.1 \pm 4.1$ & $9.4 \pm 3.9$ & $8.2 \pm 4.3$ & NS \\
\hline Age at epilepsy onset & $11.7 \pm 7.9$ & $13.1 \pm 7.9$ & $19.2 \pm 15.2$ & NS \\
\hline Epilepsy duration & $31.7 \pm 13.0$ & $21.8 \pm 8.1$ & $21.2 \pm 11.6$ & $<0.001^{a}$ \\
\hline Three-month seizures & $6.5 \pm 13.4$ & $1.3 \pm 2.5$ & $10.8 \pm 24.9$ & $<0.001^{\mathrm{b}}$ \\
\hline Number of Medications & $2.2 \pm 0.8$ & $1.8 \pm 1.0$ & $1.9 \pm 1.0$ & NS \\
\hline Gender (\% male) & $17(30.9 \%)$ & $10(38.5 \%)$ & $28(73.7 \%)$ & $0.002^{a}, 0.005^{c}$ \\
\hline \multicolumn{5}{|c|}{ Socioeconomic classification } \\
\hline A or $B$ & $11(20.0 \%)$ & $12(48.0 \%)$ & $10(26.3 \%)$ & NS \\
\hline C & $33(60.0 \%)$ & $12(48.0 \%)$ & $25(65.8 \%)$ & NS \\
\hline Dor $E$ & $11(20.0 \%)$ & $1(4.0 \%)$ & $3(7.9 \%)$ & NS \\
\hline History of febrile seizure & $13(23.6 \%)$ & $1(3.8 \%)$ & $2(5.3 \%)$ & $0.003^{a}$ \\
\hline
\end{tabular}

MTS: Mesial temporal sclerosis group; PGE: Primary generalized epilepsy group; FSE: Focal symptomatic epilepsy group; NC: Neurocysticercosis; NS: statistically non-significant; Patients were classified in one of five socioeconomic classes (A to E) based on a socioeconomic questionnaire. One patient in the IGE group did not fill out the socioeconomic questionnaire. Between groups statistical comparison: ${ }^{\mathrm{a} M T S}$ vS. FSE and PGE; ${ }^{\mathrm{b} M T S}$ vs. PGE; ${ }^{\mathrm{C}} \mathrm{PGE}$ vs. FSE. 


\begin{tabular}{|c|c|c|c|}
\hline & $\begin{array}{l}\text { With NC } \\
(n=15)\end{array}$ & $\begin{array}{l}\text { Without NC } \\
\quad(n=40)\end{array}$ & $p$ value \\
\hline Age & $46.6 \pm 10.0$ & $42.2 \pm 12.7$ & NS \\
\hline Education & $7.2 \pm 3.9$ & $7.1 \pm 4.2$ & NS \\
\hline Age at epilepsy onset & $8.3 \pm 5.9$ & $13.0 \pm 8.3$ & NS \\
\hline Epilepsy duration & $38.4 \pm 10.2$ & $29.3 \pm 13.1$ & 0.021 \\
\hline Number of seizures & $6.4 \pm 11.4$ & $6.5 \pm 14.2$ & NS \\
\hline Medication number & $2.1 \pm 0.9$ & $2.2 \pm 0.7$ & NS \\
\hline Male gender & $5(33.3 \%)$ & $12(30.0 \%)$ & NS \\
\hline \multicolumn{4}{|l|}{ Socioeconomic classification } \\
\hline$A$ or $B$ & $1(6.7 \%)$ & $10(25.0 \%)$ & NS \\
\hline C & $12(80.0 \%)$ & $21(52.5 \%)$ & NS \\
\hline $\mathrm{D}$ or $\mathrm{E}$ & $2(13.3 \%)$ & $9(22.5 \%)$ & NS \\
\hline Initial precipitating insult/ Febrile seizure & $4(26.7 \%)$ & $9(22.5 \%)$ & NS \\
\hline Previous history of living in rural areas & $13(86.7 \%)$ & $21(52.5 \%)$ & 0.029 \\
\hline Previous contact with pigs & $14(93.3 \%)$ & $19(47.5 \%)$ & 0.004 \\
\hline
\end{tabular}

MTS: Mesial temporal sclerosis group; NC: Neurocysticercosis; NS: statistically non-significant. Patients were classified in one of five socioeconomic classes ( $A$ to $E$ ) based on a socioeconomic questionnaire.

suggestive of NC on brain CT in MTS patients in endemic regions ${ }^{11,12}$. This could be a fortuitous finding. NC and MTS could conceivably occur in conjunction with lower socioeconomic and sanitation conditions, patients living in endemic regions, and contact with pigs. In this scenario presence of NC in MTS could represent a confounding factor.

Using single or multiple small calcified lesions as a surrogate marker for inactive neurocysticercosis may also present limitations. Solitary and multiple small calcified lesions are not specific findings of neurocysticercosis, and may be also related to other granulomatous etiologies ${ }^{14}$. In endemic areas, typical small calcified lesions are usually interpreted as highly suggestive of inactive NC. The diagnosis of definite $\mathrm{NC}$ is established by presence of one absolute criterium or of two major plus one minor and one epidemiologic criteria ${ }^{15}$. All absolute criteria require the presence of the parasite in the active form (i.e. lesions with scolex). Other criteria include serologic and evolutive evidence of NC, that may be absent in patients with inactive NC. Brain calcifications suggestive of neurocysticercosis are major criteria. Our patients do not fulfill definite NC criteria, but they can be classified as probable NC, with one major (brain calcifications), one minor (seizures) and one epidemiological (NC endemic area) criterium. Using the 2002 proposed diagnostic criteria ${ }^{15}$ in this study would be limited, since these criteria were established as a diagnostic tool for active neurocysticercosis.

In population-based studies in endemic regions, 10 to $18 \%$ of asymptomatic individuals have CT findings suggestive of neurocysticercosis ${ }^{16}$. In our study, small calcified lesions, interpreted as suggestive of inactive $\mathrm{NC}$, were found in $27.5 \%$ of MTS patients, in a significantly higher proportion than in the other epilepsy types. The frequency of inactive NC in our MTS patients was similar to that encountered in a study performed in another tertiary referral hospital in a different town in Sao Paulo State, Brazil ${ }^{11}$.

In this study we randomly selected patients from three different epilepsy types, independently of the presence of NC lesions. Prevalence of inactive NC was significantly higher in the MTS, than in PGE and FSE groups. The clinical and demographic features of the different epilepsy types are consistent with well recognized characteristics of these syndromes. Patients with MTS were also older, had longer disease duration compared to PGE patients, and, as expected, referred more commonly a previous history of an IPI. The difference in gender proportion among groups cannot be easily explained, and may be related to sampling bias.

The different number of cases included in each epilepsy group may raise concern for possible selection bias. This possibility cannot be excluded. However, we do not believe this was the case. Case selection was independent of presence of NC calcifications. Unequal group sizes probably reflect different prevalences of the selected syndromes in a tertiary hospital setting. The highest proportion of MTS patients is not surprising in an epilepsy outpatient clinic based in a major teaching hospital, in a country with relatively few epilepsy surgery centers. The size of the PGE patients group is consistent with the prevalence of this epilepsy syndrome.

Another potential source of bias is the fact that some interviewed patients did not undergo a head CT. We do not believe a selection bias occurred. A similar proportion of patients belonging to the three groups did not undergo a head CT. The only difference between the group of patients that underwent a head CT and those who did not, is that patients who underwent a head CT were older and had presented more seizures in the previous three months, compared to the patients who did not show up for the CT appointment. It is conceivable that patients 
with more severe illness tend to be adherent to scheduled tests and appointments. This fact should not impact on a higher proportion of MTS patients with NC undergoing a head CT.

On multivariate analysis, we found that presence of one or more calcified NC lesions was independently associated with finding of MTS on MRI, as well as to a report of previous contact with pigs. Patients with MTS had a threefold higher chance of presenting small calcified lesions compared to patients with other epilepsy types. Although the prevalence of calcified lesions in the other groups is relatively high (8\%), we found an approximately $20 \%$ excess prevalence of calcified lesions in the MTS group. After controlling for possible confounding factors, these data show an independent association between one or more calcified lesions, suggestive of cysticercosis, and MTS.

Not all calcified lesions seen on head CT are necessarily due to NC (i.e. most inflammatory granulomas will heal as calcifications). In endemic areas, the majority of calcifications are believed to be NC-related ${ }^{16}$. Independent association between one or more calcified lesions and a history of contact with pigs further strengthen the possibility that the punctate calcified lesions seen on CT are indeed related to NC. Contact with pigs and a history of living (or having lived) in rural areas are not mandatory for the diagnosis of neurocysticercosis. Cases of neurocysticercosis have been reported in orthodox Jews in New York City, traced to contamination from a Central American house servant, and in other nonendemic areas ${ }^{17}$. However, living in rural areas, having domestic contact with pigs and harboring intestinal infection with Taenia solium increase the risk of recurrent infestation with eggs of the parasite, and of acquiring neurocysticercosis ${ }^{16}$.

Association of MTS and calcified lesions was only observed considering one or more calcified lesions suggestive of NC. This association did not reach statistical significance on multivariate analysis, considering the finding of two or more calcified lesions. This is probably due to small sample size of patients harboring two or more calcifications. From a pathophysiological standpoint, the finding of one or more lesions may be irrelevant for the association of NC and MTS. Other authors have speculated that inflammatory responses to the cysticercus may be related to the emergence of $\mathrm{MTS}^{6}$. If this is the case, the presence of one or more lesions may not make a difference in the disease generating process.

Although numbers are small, hindering statistical analysis, we found a higher mean number of calcifications in the MTS group compared with other epilepsy groups. Also, in the MTS group we also found a greater proportion of lesions in the temporal lobe ipsilateral to the MTS.

Comparing epilepsy features of MTS patients with and without calcified lesions concerning epilepsy features, we did not find differences between the two groups, except for the fact that patients with calcified lesions had longer epilepsy duration. We did not find a difference in prevalence of reported IPI in the two groups, The possibility that NC may act as an IPI would, therefore, not be supported by our findings. One could argue that NC may trigger the IPI (febrile seizure) in the NC-MTS group. This remains speculative and this hypothesis should be further evaluated.

This study format does not allow us to establish a causeeffect relationship between NC and MTS. Mechanisms involved in MTS and NC association should be further investigated in another study design, such as a populationbased study.

The findings of the present study lend further support to the notion that hippocampal sclerosis (HS) should be included in the spectrum of the clinical manifestations of $\mathrm{NC}$-associated epilepsy. If a causal relationship between NC and MTS is established, improvements in sanitation and meat inspection may be effective to decrease prevalence of epilepsy associated with HS in endemic areas, a significant cause of medically refractory epilepsy with cognitive and psychiatric comorbidities, and negative impact on quality of life in endemic regions.

\section{References}

1. Cendes F. Febrile seizures and mesial temporal sclerosis. Curr Opin Neurol 2004;17:161-164.

2. Hirose S, Mohney RP, Okada M, Kaneko S, Mitsudome A. The genetics of febrile seizures and related epilepsy syndromes. Brain Dev 2003;25:304-312.

3. Nash TE, Del Brutto OH, Butman JA, et al. Calcific neurocysticercosis and epileptogenesis. Neurology 2004;62:1934-1938.

4. Nash TE, Singh G, White AC, et al. Treatment of neurocysticercosis: current status and future research needs. Neurology 2006;67:1120-1127.

5. Leite JP, Terra-Bustamante VC, Fernandes RM, et al. Calcified neurocysticercotic lesions and postsurgery seizure control in temporal lobe epilepsy. Neurology 2000;55:1485-1491.
6. Bianchin MM, Velasco TR, Takayanagui OM, Sakamoto AC Neurocysticercosis, mesial temporal lobe epilepsy, and hippocampal sclerosis: an association largely ignored. Lancet Neurol 2006;5:20-21.

7. Bianchin MM, Velasco TR, Wichert-Ana L, Takayanagui OM, Leite JP, Sakamoto AC. How frequent is the association of neurocysticercosis and mesial temporal lobe epilepsy with hippocampal sclerosis? Epilepsia 2010;51:2359-2360.

8. Kobayashi E, Guerreiro CA, Cendes F. Late onset temporal lobe epilepsy with MRI evidence of mesial temporal sclerosis following acute neurocysticercosis: case report. Arq Neuropsiquiatr 2001;59:255-258.

9. da Silva AV, Martins HH, Marques CM, et al. Neurocysticercosis and microscopic hippocampal dysplasia in a patient with refractory mesial temporal lobe epilepsy. Arq Neuropsiquiatr 2006;64:309-313. 
10. Singla M, Singh P, Kaushal S, Bansal R, Singh G. Hippocampal sclerosis in association with neurocysticercosis. Epileptic Disord 2007;9:292-299.

11. Velasco TR, Zanello PA, Dalmagro CL, et al. Calcified cysticercotic lesions and intractable epilepsy: a cross sectional study of 512 patients. J Neurol Neurosurg Psychiatry 2006;77:485-488.

12. da Gama CN, Kobayashi E, Li LM, Cendes F. Hippocampal atrophy and neurocysticercosis calcifications. Seizure 2005;14:85-88.

13. ABEP [Brazilian Association of Research Companies]. [Internet] Critério Padrão de Classificação Econômica Brasil/2008. Available in: http://www.abep.org. Portuguese.
14. Bromfield EB, Vonsattel JP. Case records of the Massachusetts General Hospital. Weekly clinicopathological exercises. Case 242000. A 23-year-old man with seizures and a lesion in the left temporal lobe. N Engl J Med 2000;343:420-427.

15. Del Brutto OH, Rajshekhar V, White AC Jr, et al. Proposed diagnostic criteria for neurocysticercosis. Neurology 2001;57:177-183.

16. Cysticercosis Working Group in Peru. Taenia solium cysticercosis. Lancet 2003;362:547-556.

17. Schantz PM, Moore AC, Muñoz JL, et al. Neurocysticercosis in an Orthodox Jewish community in New York City. N Engl J Med 1992;327:692-695. 